Tôhoku Math. Journ.

24 (1972), 309-318.

\title{
ABSOLUTE NÖRLUND SUMMABILITY OF FOURIER SERIES
}

Dedicated to Professor Gen-ichirô Sunouchi on his 60th birthday

\section{S. M. $\mathrm{SHAH}^{1}$}

(Received Dec. 13, 1971)

1. Introduction. Let $\left\{p_{n}\right\}_{0}^{\infty}$ be a sequence of non-negative constants, $p_{0}>0$ and $P_{n}=\sum_{0}^{n} p_{k}$. A sequence $\left\{U_{n}\right\}_{0}^{\infty}$ will be said to be absolutely summable by the Nörlund method defined by the sequence $\left\{p_{n}\right\}$, or summable $\left|N, p_{n}\right|$, if $t_{n}=\sum_{\nu=0}^{n}\left(p_{n-\nu} U_{\nu}\right) / P_{n}$ and

$$
\sum_{n-1}^{\infty}\left|t_{n}-t_{n-1}\right| \leqq c<\infty \text {. }
$$

Varshney [10] showed that if $f(x)$ is a real-valued, $2 \pi$-periodic function and of bounded variation over $[0,2 \pi]$ and if

$$
|f(x+h)-f(x)| \leqq A \log ^{-1-\varepsilon}\left(\frac{1}{h}\right)(\varepsilon>0,0 \leqq x \leqq 2 \pi, h>0)
$$

then $S(f)$, the Fourier series of $f$, is summable $|N, 1 /(n+1)|$. The author [8] later proved this result under the following weaker hypothesis:

$$
\sum_{1}^{\infty} \frac{1}{n} \omega\left(\frac{1}{n}\right)<\infty,
$$

where $\omega(t, f)=\omega(t)$ denotes, as usual, the modulus of continuity of $f$. Recently Izumi and Izumi [3], Lal [5] and others have studied the conditions for $\left|N, p_{n}\right|$ summability of $S(f)$ for general $\left\{p_{n}\right\}$. Lal has shown that, if (i) $p_{0}>0$, (ii) $\left\{p_{n}\right\}$ is non-negative and non-increasing, (iii) $\lim _{n \rightarrow \infty} p_{n}=0$, (iv) $\left\{p_{n}-p_{n+1}\right\}$ is non-increasing, and if

$$
\sum_{1}^{\infty} p_{n}^{r} n^{r-2}<\infty \quad(1<r \leqq 2),
$$

and

$$
\sum_{1}^{\infty} \omega\left(n^{-1}\right) P_{n}^{-1} n^{-1 / s}<\infty,\left(\frac{1}{r}+\frac{1}{s}=1\right)
$$

then $S(f)$ is summable $\left|N, p_{n}\right|$. In this paper we obtain conditions for $\left|N, p_{n}\right|$ summability of $S(f)$ when the series in (1.4) may fail to con-

1 The research work of the author is supported by National Science Foundation Grant GP-19533. 
verge. Thus our results supplement those of Lal.

In what follows we will suppose that $\gamma$ is a fixed constant, $0 \leqq \gamma<$ $1 / 2, c_{1}$ and $c_{2}$ are fixed positive constants and $\psi(x)$ is positive on $[0, \infty)$ and slowly oscillating in the sense of Karamata (see [2], [4]). Let $\left\{p_{n}\right\}$ satisfy conditions (i)-(iv) and suppose that for $n \geqq 1$,

$$
c_{1} n^{r} \psi(n) \leqq P_{n} \leqq c_{2} n^{r} \psi(n)
$$

These conditions are all satisfied if, for instance, we take $p_{n}=(n+1)^{-1+\gamma}$, $0 \leqq \gamma<1 / 2$. Some further examples are given in Section 4 . We prove the following

THEOREM 1. Let $f(x)$ be a $2 \pi$-periodic function of bounded variation over $[0,2 \pi]$ and suppose that the modulus of continuity $\omega(t, f)$ satisfies (1.3) and

$$
\sum_{n=1}^{\infty} \frac{1}{n P_{n}} \omega^{1 / 2}\left(\frac{1}{n}\right)<\infty .
$$

Then under the assumptions (i)-(v), $S(f)$ is summable $\left|N, p_{n}\right|$.

2. Lemmas. We shall denote by $A$ a positive constant (possibly depending on $\gamma, c_{1}, c_{2}$ ) not necessarily the same at each occurence.

LEMma 1 [6]. If $\left\{p_{n}\right\}$ is non-negative and non-increasing, then for $0 \leqq a \leqq b \leqq \infty, 0 \leqq t \leqq \pi$ and any $n$, we have

$$
\left|\sum_{k=a}^{b} p_{k} e^{i(n-k) t}\right| \leqq \begin{cases}P\left(t^{-1}\right) & \text { for any } a, \\ A t^{-1} p_{[a]} & \text { for } a \geqq\left[t^{-1}\right] .\end{cases}
$$

Here $[x]$ denotes the integer part of $x$, and $P(x)=P_{[x]}$.

Lemma 2 [6]. If $\left\{p_{n}\right\}$ is non-negative and non-increasing and $\left\{p_{n}-p_{n+1}\right\}$ is non-increasing, then

$$
\frac{n^{2}\left(p_{n}-p_{n+1}\right)}{P(n-1)} \leqq \frac{n^{2}\left(p_{n-1}-p_{n}\right)}{P(n-1)} \leqq A .
$$

Lemma 3. If $P(x)$ satisfies (v) then

$$
\frac{n}{P(n-1)} \int_{n}^{\infty} \frac{P(u) d u}{u^{2}}<A
$$

This follows from the properties of slowly oscillating functions [2]. We have $\int_{n}^{\infty} u^{\gamma-2} \psi(u) d u \sim \psi(n)\left(n^{\gamma-1} / 1-\gamma\right)$, and $\psi(n) \sim \psi(n-1)$ and (2.3) follows. 
LEMMA 4. Let

$$
I_{n}=\int_{1 / \pi}^{\left(2^{n+1}\right) / \pi} \omega^{2}\left(\frac{1}{t}\right) d t .
$$

The series in (1.3) and the series

$$
\sum_{n=1}^{\infty} 2^{-n / 2} I_{n}^{1 / 2}
$$

are both convergent or both divergent.

Proof. Since

$$
I_{n}>\frac{1}{4} 2^{n} \omega^{2}\left(\frac{1}{2^{n}}\right)
$$

the convergence of (2.5) implies the convergence of $\sum_{n=1}^{\infty} \omega\left(1 / 2^{n}\right)$ and hence that of the series in (1.3). Suppose now that the series in (1.3) is convergent. Then

$$
\begin{gathered}
I_{n}<\omega^{2}(\pi)+\omega^{2}(1)+2 \omega^{2}\left(\frac{1}{2}\right)+\cdots+2^{n-1} \omega^{2}\left(\frac{1}{2^{n-1}}\right) \\
\sum_{n=1}^{\infty} 2^{-n / 2} I_{n}^{1 / 2}<\omega(\pi) \sum_{1}^{\infty} 2^{-n / 2}+\sum_{n=1}^{\infty} 2^{-n / 2} \sum_{p=1}^{n-1} 2^{p / 2} \omega\left(\frac{1}{2^{p}}\right) \\
<A+\sum_{p=1}^{\infty} 2^{p / 2} \omega\left(\frac{1}{2^{p}}\right) \sum_{n=p+1}^{\infty} 2^{-n / 2} \\
<A+A \sum_{p=1}^{\infty} \omega\left(\frac{1}{2^{p}}\right)<A .
\end{gathered}
$$

3. Proof of Theorem 1. Let

$$
\begin{gathered}
f(t) \sim \frac{1}{2} a_{0}+\sum_{1}^{\infty}\left(a_{n} \cos n t+b_{n} \sin n t\right) \equiv \sum_{0}^{\infty} u_{n}, \\
s_{n}=\sum_{\nu=0}^{n} u_{\nu}, t_{n}=\sum_{\nu=0}^{n} \frac{p_{n} s_{n-\nu}}{P_{n}}, \\
\phi(t)=f(x+t)+f(x-t)-2 f(x), \\
\alpha(t)+i \beta(t)=\sum_{k=0}^{\infty} p_{k} e^{i k t}, \\
\alpha_{n}=\int_{0}^{\pi} \phi(t) \alpha(t) \cos n t d t, \quad \beta_{n}=\int_{0}^{\pi} \phi(t) \beta(t) \sin n t d t .
\end{gathered}
$$

We have (cf: [6], [8]) 


$$
\begin{aligned}
\pi\left|t_{n}-t_{n-1}\right|= & \left|\int_{0}^{\pi} \phi(t) \sum_{k=0}^{n-1}\left(\frac{P_{k}}{P_{n}}-\frac{P_{k-1}}{P_{n-1}}\right) \cos (n-k) t d t\right| \\
\leqq & \frac{1}{P_{n-1}}\left|\int_{0}^{\pi} \phi(t) \sum_{k=0}^{\infty} p_{k} \cos (n-k) t d t\right| \\
& +\frac{1}{P_{n-1}}\left|\int_{0}^{1 / n} \phi(t) \sum_{k=n}^{\infty} p_{k} \cos (n-k) t d t\right| \\
& +\frac{p_{n}}{P_{n} P_{n-1}}\left|\int_{0}^{1 / n} \phi(t) \sum_{k=0}^{n-1} P_{k} \cos (n-k) t d t\right| \\
& +\frac{1}{P_{n-1}}\left|\int_{1 / n}^{\pi} \phi(t)\left\{\sum_{k=n}^{\infty} p_{k} \cos (n-k) t+\sum_{k=0}^{n-1} \frac{p_{n}}{P_{n}} P_{k} \cos (n-k) t\right\} d t\right| \\
= & T_{1}(n)+T_{2}(n)+T_{3}(n)+T_{4}(n) \text { say } .
\end{aligned}
$$

We have to prove that $\sum\left|t_{n}-t_{n-1}\right|<\infty$. By Lemmas 1 and 3

$$
T_{2}(n)<\frac{2}{P_{n-1}} \int_{0}^{1 / n} \omega(t) P\left(\frac{1}{t}\right) d t<\frac{2 \omega(1 / n)}{P_{n-1}} \int_{n}^{\infty} \frac{P(u) d u}{u^{2}}<A \frac{1}{n} \omega\left(\frac{1}{n}\right)
$$

and by (1.3), $\sum_{n=2}^{\infty} T_{2}(n)<\infty$.

Further, since $p_{n} \downarrow$,

$$
\begin{aligned}
T_{3}(n) & <\frac{2 p_{n}}{P_{n} P_{n-1}} \omega\left(\frac{1}{n}\right) \frac{P_{0}+\cdots+P_{n-1}}{n} \\
& <\frac{2 p_{n}}{P_{n} P_{n-1}} \omega\left(\frac{1}{n}\right) P_{n-1}<\frac{2}{n} \omega\left(\frac{1}{n}\right)
\end{aligned}
$$

and so $\sum_{n=2}^{\infty} T_{3}(n)<\infty$.

Further

$$
\begin{aligned}
T_{4}(n)= & \frac{1}{P_{n-1}} \mid \int_{1 / n}^{\pi} \phi(t)\left[\frac{p_{n}}{2}+\sum_{k=n}^{\infty}\left(p_{k}-p_{k+1}\right) \frac{\sin (n-k+(1 / 2)) t}{2 \sin (t / 2)}\right. \\
& \left.+\frac{p_{n}}{P_{n}}\left\{\sum_{k=0}^{n-1} p_{k} \frac{\sin (n-k+(1 / 2)) t}{2 \sin (t / 2)}-\frac{1}{2} P_{n-1}\right\}\right] d t \mid \\
\leqq & \frac{1}{P_{n-1}}\left|\int_{1 / n}^{\pi} \frac{\phi(t)}{2 \sin (t / 2)}\left(\sum_{k=n}^{\infty}\left(p_{k}-p_{k+1}\right) \sin \left(n-k+\frac{1}{2}\right) t\right) d t\right| \\
& +\frac{p_{n}}{P_{n} P_{n-1}}\left|\int_{1 / n}^{\pi} \frac{\phi(t)}{2 \sin (t / 2)}\left(\sum_{k=0}^{n-1} p_{k} \sin \left(n-k+\frac{1}{2}\right) t\right) d t\right| \\
& +\frac{p_{n}}{2 P_{n-1}}\left(1-\frac{P_{n-1}}{P_{n}}\right)\left|\int_{1 / n}^{\pi} \phi(t) d t\right| \\
\equiv & T_{41}(n)+T_{42}(n)+T_{43}(n) .
\end{aligned}
$$

By Lemma 1 


$$
\begin{aligned}
T_{41}(n) & \leqq \frac{A\left(p_{n}-p_{n+1}\right)}{P_{n-1}} \int_{1 / n}^{\pi} \frac{|\phi(t)|}{\sin (t / 2)} t^{-1} d t \\
& \leqq \frac{A\left(p_{n}-p_{n+1}\right)}{P_{n-1}} \int_{1 / n}^{\pi} \frac{\omega(t)}{t^{2}} d t \\
& \leqq \frac{A\left(p_{n}-p_{n+1}\right)}{P_{n-1}}\left(A+\sum_{k=2}^{n} \omega\left(\frac{1}{k}\right)\right)
\end{aligned}
$$

Lemma 2 now shows that

$$
\begin{aligned}
\sum_{n=2}^{\infty} T_{41}(n) & \leqq A \sum_{n=2}^{\infty} \frac{1}{n^{2}}\left(A+\sum_{k=2}^{n} \omega\left(\frac{1}{k}\right)\right)<A+A \sum_{k=2}^{\infty} \omega\left(\frac{1}{k}\right) \sum_{n=k}^{\infty} \frac{1}{n^{2}} \\
& <A+A \sum_{k=2}^{\infty} \frac{1}{k} \omega\left(\frac{1}{k}\right)<\infty .
\end{aligned}
$$

Further $\int_{1 / n}^{\pi}|\phi(t)| d t<A$ and so

$$
\sum_{n=2}^{\infty} T_{43}(n)<A \sum_{2}^{\infty} \frac{p_{n}^{2}}{P_{n} P_{n-1}}<A \sum_{2}^{\infty} \frac{p_{n}^{2}}{(n+1) p_{n} n p_{n-1}}<A \sum_{2}^{\infty} \frac{1}{n^{2}}<\infty .
$$

By Lemma 1

$$
T_{42}(n) \leqq \frac{A p_{n}}{P_{n} P_{n-1}} \int_{1 / n}^{\pi} \frac{|\phi(t)|}{t} P\left(\frac{1}{t}\right) d t \leqq \frac{A p_{n}}{P_{n} P_{n-1}} \int_{1 / \pi}^{n} \omega\left(\frac{1}{t}\right) P(t) \frac{d t}{t},
$$

and

$$
\begin{aligned}
\sum_{n=2}^{\infty} T_{42}(n) & \leqq A \sum_{n=2}^{\infty} \frac{p_{n}}{P_{n} P_{n-1}}\left(A+\sum_{1}^{n} \omega\left(\frac{1}{k}\right) \frac{P(k)}{k}\right) \\
& <A+A \sum_{n=2}^{\infty} \frac{p_{n}}{P_{n} P_{n-1}} \sum_{k=1}^{n} \omega\left(\frac{1}{k}\right) \frac{P(k)}{k} \\
& =A+A \sum_{k=1}^{\infty} \omega\left(\frac{1}{k}\right) \frac{P(k)}{k} \sum_{n=k}^{\infty} \frac{p_{n}}{P_{n} P_{n-1}} \\
& <A+A \sum_{k=1}^{\infty} \omega\left(\frac{1}{k}\right) \frac{P(k)}{k} \frac{1}{P(k-1)} \\
& <A+A \sum_{1}^{\infty} \omega\left(\frac{1}{k}\right) \frac{1}{k}<\infty .
\end{aligned}
$$

We now consider $T_{1}(n) \leqq\left(\left|\alpha_{n}\right|+\left|\beta_{n}\right|\right) / P_{n-1}$.

Let $\Psi(t)=\phi(t+h) \alpha(t+h)-\phi(t-h) \alpha(t-h)$. Then $\Psi(t)$ is even and $\alpha(t) \in L^{2}$, for by Lemma 1 ,

$$
\int_{0}^{\pi} \alpha^{2}(t) d t \leqq \int_{0}^{\pi} P^{2}\left(\frac{1}{t}\right) d t<A \int_{0}^{\pi} \psi^{2}\left(\frac{1}{t}\right) t^{-2 \gamma} d t<\infty .
$$


By Bessel's inequality we have for $0<h \leqq \pi / 4$,

$$
\begin{aligned}
\sum_{1}^{\infty}\left|\alpha_{n}^{2} \sin ^{2} n h\right|< & A \int_{0}^{\pi}\left|\Psi^{2}(t)\right| d t \\
< & A\left[\int_{0}^{\pi} \alpha^{2}(t+h)|\{\phi(t+h)-\phi(t-h)\}|^{2} d t\right. \\
& +\int_{-h}^{h}\left|\phi^{2}(t)\right| \alpha^{2}(t+2 h) d t+\int_{-h}^{h}\left|\phi^{2}(t)\right| \alpha^{2}(t) d t \\
& \left.+\int_{h}^{\pi}\left|\phi^{2}(t)\right|\{\alpha(t+2 h)-\alpha(t)\}^{2} d t\right] \\
\equiv & A\left[I_{1}(h)+I_{2}(h)+I_{3}(h)+I_{4}(h)\right] .
\end{aligned}
$$

By (v) and Lemma 1,

$$
\begin{aligned}
I_{2}(h) & <A \int_{-h}^{h} \omega^{2}(t) P^{2}\left(\frac{1}{t+2 h}\right) d t \\
& \leqq A \omega^{2}(h) \int_{h}^{3 h} P^{2}\left(\frac{1}{t}\right) d t<A h \omega^{2}(h) P^{2}\left(\frac{1}{h}\right),
\end{aligned}
$$

and

$$
\begin{aligned}
I_{3}(h) & =\int_{-h}^{h}\left|\phi^{2}(t)\right| \alpha^{2}(t) d t<A \omega^{2}(h) \int_{-h}^{h} \alpha^{2}(t) d t \\
& <A \omega^{2}(h) \int_{0}^{h} P^{2}\left(\frac{1}{t}\right) d t<A \omega^{2}(h) h P^{2}\left(\frac{1}{h}\right) .
\end{aligned}
$$

Since [6]

$$
\begin{gathered}
|\alpha(t+2 h)-\alpha(t)| \leqq A h t^{-1} P\left(h^{-1}\right), \\
I_{4}(h)<A h^{2} P^{2}\left(\frac{1}{h}\right) \int_{h}^{\pi} \frac{\omega^{2}(t)}{t^{2}} d t<A h^{2} P^{2}\left(\frac{1}{h}\right) \int_{1 / \pi}^{1 / h} \omega^{2}\left(\frac{1}{t}\right) d t .
\end{gathered}
$$

We now estimate $I_{1}$. Since $f$ is of bounded variation over $[0,2 \pi]$ we have

$$
\sum_{k=1}^{2 N} \alpha^{2}\left(t+\frac{k \pi}{N}\right)\left|\left\{\phi\left(t+\frac{k \pi}{N}\right)-\phi\left(t+(k-1) \frac{\pi}{N}\right)\right\}^{2}\right|<A \omega\left(\frac{\pi}{N}\right) P^{2}\left(\frac{1}{t}\right) .
$$

Integrating from 0 to $\pi$ (cf. [8; p. 241-2]) we get

$$
2 N I_{1}\left(\frac{\pi}{2 N}\right)<A \omega\left(\frac{\pi}{N}\right) \int_{0}^{\pi} P^{2}\left(\frac{1}{t}\right) d t<A \omega\left(\frac{\pi}{N}\right) \int_{1 / \pi}^{\infty} \frac{P^{2}(t) d t}{t^{2}}<A \omega\left(\frac{\pi}{N}\right) .
$$

Taking $h=\pi /(2 N)$ we get 


$$
\begin{aligned}
\sum_{1}^{\infty}\left|\alpha_{n}^{2} \sin ^{2}\left(\frac{n \pi}{2 N}\right)\right|< & A\left\{\frac{1}{N} \omega\left(\frac{\pi}{N}\right)+\frac{1}{N} \omega^{2}\left(\frac{\pi}{2 N}\right) P^{2}\left(\frac{2 N}{\pi}\right)\right. \\
& \left.+\frac{1}{N^{2}} P^{2}\left(\frac{2 N}{\pi}\right) \int_{1 / \pi}^{(2 N) / \pi} \omega^{2}\left(\frac{1}{t}\right) d t\right\} \\
& <A\left\{\frac{1}{N} \omega\left(\frac{\pi}{N}\right)+\frac{1}{N^{2}} P^{2}\left(\frac{2 N}{\pi}\right) \int_{1 / \pi}^{(2 N) / \pi} \omega^{2}\left(\frac{1}{t}\right) d t\right\}
\end{aligned}
$$

Letting $N=2^{\nu}$ we have

$$
\begin{aligned}
\left\{\sum_{2^{\nu}-1_{+1}}^{2^{\nu}}\left|\alpha_{n}^{2}\right|\right\}^{1 / 2} & <A\left\{\sum_{1}^{\infty}\left|\alpha_{n}^{2}\right| \sin ^{2}\left(\frac{n \pi}{2^{\nu+1}}\right)\right\}^{1 / 2} \\
& <A\left\{\frac{1}{2^{\nu}} \omega\left(\frac{\pi}{2^{\nu}}\right)+\frac{1}{2^{2 \nu}} P^{2}\left(\frac{2^{\nu+1}}{\pi}\right) \int_{1 / \pi}^{2^{\nu+1 / \pi}} \omega^{2}\left(\frac{1}{t}\right) d t\right\}^{1 / 2} \\
& <A\left\{\frac{1}{2^{\nu / 2}} \omega^{1 / 2}\left(\frac{\pi}{2^{\nu}}\right)+\frac{1}{2^{\nu}} P\left(\frac{2^{\nu+1}}{\pi}\right)\left(\int_{1 / \pi}^{2^{\nu+1} / \pi} \omega^{2}\left(\frac{1}{t}\right) d t\right)^{1 / 2}\right\} .
\end{aligned}
$$

By (v) we have

$$
\sum_{2^{\nu}=1_{+1}}^{2^{\nu}} \frac{1}{P_{n-1}^{2}}<A \frac{2^{\nu}}{P^{2}\left(2^{\nu}\right)}
$$

and an application of Schwarz inequality gives

$$
\sum_{2^{\nu}-1_{+1}}^{2^{\nu}} \frac{\left|\alpha_{n}\right|}{P_{n-1}} \leqq A \frac{2^{\nu / 2}}{P\left(2^{\nu}\right)}\left\{\frac{1}{2^{\nu / 2}} \omega^{1 / 2}\left(\frac{\pi}{2^{\nu}}\right)+\frac{1}{2^{\nu}} P\left(\frac{2^{\nu+1}}{\pi}\right)\left(\int_{1 / \pi}^{2^{\nu+1} / \pi} \omega^{2}\left(\frac{1}{t}\right) d t\right)^{1 / 2}\right\} .
$$

By (1.5),

$$
\sum \frac{1}{P\left(2^{\nu}\right)} \omega^{1 / 2}\left(\frac{\pi}{2^{\nu}}\right)<A
$$

and by Lemma 4 ,

$$
\sum \frac{P\left(2^{\nu+1 / \pi}\right)}{P\left(2^{\nu}\right)} \frac{1}{2^{2 / 2}}\left(\int_{1 / \pi}^{2^{\nu+1 / \pi}} \omega^{2}\left(\frac{1}{t}\right) d t\right)^{1 / 2}<A .
$$

Hence $\sum_{n=1}^{\infty}\left|\alpha_{n}\right| / P_{n-1}<\infty$. Similarly $\sum_{n=1}^{\infty}\left|\beta_{n}\right| / P_{n-1}<\infty$ and so $\sum_{2}^{\infty}\left|t_{n}-t_{n-1}\right|<$ $A<\infty$ and the proof is complete.

4. Remarks and Examples.

(a) If (1.3) holds and $\sum 1 /\left(n P^{2}(n)\right)<\infty$, then an application of Schwarz inequality shows that (1.5) holds.

(b) The condition (1.5) implies that

$$
\omega\left(\frac{1}{t}\right)<A\left(P^{2}(t)\right) / \log ^{2} t, \quad t \geqq 2 .
$$

Consequently 


$$
\sum \omega\left(\frac{1}{2^{n}}\right)<A \sum\left(P^{2}\left(2^{n}\right)\right) / n^{2}
$$

Hence if (1.5) holds and

$$
\sum\left(P^{2}\left(2^{n}\right)\right) / n^{2}<\infty,
$$

then the series in (1.3) is convergent.

If we take, for instance, $p_{n}=(n+a)^{-1}(\log (n+a))^{-1}, a \geqq 3$, then by considering $y(x)=(x+a)^{-1}(\log (x+a))^{-1}$ we see that $p_{n}$ satisfies the conditions (i)-(iv). Further $P_{n} \sim \log \log n$ and so (4.1) and (v) are satisfied (with $\gamma=0$ ). and

(c) Zygmund $[11 ; 241-2]$ proved that if $f(x)$ is of bounded variation

$$
\sum n^{-1} \omega^{1 / 2}\left(n^{-1}\right)<\infty,
$$

then $S(f)$ is absolutely convergent. Our theorem gives the following analogue of Zygmund's result:

If $f(x)$ is of bounded variation and if (4.1) holds, the then convergence of the series in (1.5) implies the absolute summability $\left|N, p_{n}\right|$ of $S(f)$.

Note that if we take $p_{0}=1$ and $p_{n}=0 \quad(n>0)$ then (1.5) is the same as (4.2) and the summability $\left|N, p_{n}\right|$ is the same as the absolute convergence.

Example. Let

$$
p_{n}=\frac{c \log (n+c)}{(n+c) \log c}, \quad \log c \geqq 2 .
$$

Then $p_{n}>0,\left\{p_{n}\right\} \downarrow,\left\{p_{n}-p_{n+1}\right\} \downarrow$ (cf: [6]). $P_{n} \sim A(\log n)^{2}$. Hence condition (v) is satisfied (with $\gamma=0$ ) and $\sum 1 /\left(n P_{n}^{2}\right)<\infty$. (This implies that (1.5) is satisfied if (1.3) is.) By considering $y^{\prime}(x)$ where

$$
y(x)=\frac{(x+c)}{(x+1+c)} \frac{\log (x+1+c)}{\log (x+c)},
$$

we see that $p_{n+1} / p_{n} \uparrow$ and so by a known inclusion theorem [6], $\left|N, p_{n}\right| \subset$ $|C, 1|$.

5. Weighted Arithmetic Means. We now consider the weighted arithmetic mean ([7; pp. 16-17], [9; p. 32]) of the series $\sum_{0}^{\infty} u_{n}$. Let $S_{k}=\sum_{0}^{k} u_{n}$. Let $p_{n} \geqq 0, P_{n}>0$ and $\sigma_{n}=1 / P_{n} \sum_{k=0}^{n} p_{k} S_{k}$. To avoid trivial cases we shall suppose that $p_{n}>0$ for an infinity of $n$. The sequence $\left\{S_{k}\right\}$ is said to be absolutely summable by the weighted arithmetic mean method, defined by the sequence $\left\{p_{n}\right\}$, or briefly summable $\left|M, p_{n}\right|$, if 


$$
\sum_{n=1}^{\infty}\left|\sigma_{n}-\sigma_{n-1}\right| \leqq A<\infty
$$

Let $f \in C_{2 \pi}$ (continuous and $2 \pi$-periodic) and let

$$
\omega_{2}(\delta, f)=\sup _{0 \leqq h \leqq \delta}|f(x+h)+f(x-h)-2 f(x)|(x \in[0,2 \pi])
$$

denote the modulus of smoothness of $f$.

Theorem 2. Let $p_{n} \geqq 0, P_{n}=\sum_{0}^{n} p_{j}>0, P_{n} \rightarrow \infty$ and $f \in C_{2 \pi}$. If

$$
\sum_{n=1}^{\infty} \frac{p_{n}}{P_{n}} \log n \omega_{2}\left(\frac{1}{n}\right)<\infty,
$$

then $S(f)$ is summable $\left|M, p_{n}\right|$.

Proof. We have $[1 ;$ p. 300, p. 533]

$$
\left|S_{n}(t)-f(t)\right|<C \omega_{2}\left((n+1)^{-1}\right) \max (1, \log n)
$$

where $C$ is an absolute constant. Hence for $n \geqq 1$,

$$
\begin{aligned}
\left|\sigma_{n}(t)-\sigma_{n-1}(t)\right| & =\left|\frac{1}{P_{n}} \sum_{0}^{n} p_{k}\left(S_{k}(t)-f(t)\right)-\frac{1}{P_{n-1}} \sum_{0}^{n-1} p_{k}\left(S_{k}(t)-f(t)\right)\right| \\
& =\left|\left(\frac{1}{P_{n}}-\frac{1}{P_{n-1}}\right) \sum_{0}^{n-1} p_{k}\left(S_{k}(t)-f(t)\right)+\left(\frac{1}{P_{n}}\right) p_{n}\left(S_{n}(t)-f(t)\right)\right| .
\end{aligned}
$$

Thus for $0 \leqq t \leqq 2 \pi$,

$$
\begin{aligned}
\sum_{n=1}^{\infty}\left|\sigma_{n}(t)-\sigma_{n-1}(t)\right| \leqq & C \sum_{n=1}^{\infty}\left(\frac{1}{P_{n-1}}-\frac{1}{P_{n}}\right) \sum_{k=0}^{n-1} p_{k} \omega_{2}\left(\frac{1}{k+1}\right) \max (1, \log k) \\
& +C \sum_{n=1}^{\infty} \frac{p_{n}}{P_{n}} \omega_{2}\left(\frac{1}{n+1}\right) \max (1, \log n) \\
\leqq & 2 C\left\{\sum_{k=0}^{\infty} \frac{p_{k}}{P_{k}} \omega_{2}\left(\frac{1}{k+1}\right) \max (1, \log k)\right\},
\end{aligned}
$$

and our hypothesis shows that the last series is convergent. The proof is complete.

CoRollary. If $f \in C_{2 \pi}$ and $\sum_{1}^{\infty}(\log n /(n+1)) \omega_{2}(1 / n)<\infty$, then $S(f)$ is summable $|C, 1|$.

\section{REFERENCES}

[1] N. K. Bary, A Treatise on Trigonometric Series, Vol. 1, Pergamon, New York, 1964.

[2] G. H. Hardy and W. W. Rogosinski, Note on Fourier series (III): Asymptotic formulae for the sums of certain trigonometrical series, Quarterly J. of Math. (Oxford), 16 (1945), 49-58.

[3] M. Izumi and S. Izumi, Nörlund Summability of Fourier series, Pacific J. Math. 26 
(1968), 289-301.

[4] J. Karamata, Sur un mode de croissance reguliere, Bull. Soc. Math. France 61 (1933), 55-62.

[ 5 ] S. N. LAL, On a theorem of Izumi and Izumi, Pacific J. Math. 35 (1970), 661-667.

[6] L. McFadden, Absolute Nörlund Summability, Duke Math. J. 9 (1942), 1968-207.

[7] A. Peyerimhoff, Lectures on Summability, Lecture notes in mathematics, Vol. 107, Springer, New York, 1969.

[8] S. M. SHAH, On the absolute harmonic summability of Fourier series, Proc. Amer. Math. Soc. 13 (1962), 244-250.

[9] O. Szasz, Introduction to the Theory of Divergent Series, Stechert-Hafner, New York, 1952.

[10] O. P. Varshney, On the absolute harmonic summability of Fourier series, Proc. Amer. Math. Soc. 11 (1960), 588-595.

[11] A. Zygmund, Trigonometric Series, Vol. 1, Cambridge Univ. Press, New York, 1959.

UNIVERSITY OF KENTUCKY

Lexington, Kentucky 40506, U.S.A. 